\title{
4-Hydroxy-2'-Nitrodiphenyl Ether Analogues as Novel Tyrosinase Inhibitors
}

\author{
Kiran Sapkota, Eunyoung Lee, ${ }^{\dagger}$ Jae-Ho Yang, ${ }^{\ddagger}$ Youngjoo Kwon, ${ }^{\dagger}$ Jongwon Choi, ${ }^{\S}$ and Younghwa Na ${ }^{*}$ \\ College of Pharmacy, Catholic University of Daegu, Gyeongsan, Gyeongbuk, Korea."E-mail: yna7315@cu.ac.kr \\ ${ }^{\dagger}$ College of Pharmacy, Ewha Womans University, Seoul 120-750, Korea \\ ${ }^{\ddagger}$ The Marine Biotechnology Research Center, Catholic University of Daegu, Gyeongsan, Gyeongbuk, Korea \\ ${ }^{\S}$ College of Pharmacy, Kyungsung University, Pusan, Korea \\ Received February 16, 2010, Accepted March 17, 2010
}

\begin{abstract}
Tyrosinase ubiquitously existing from microorganisms to animals and plants is known to be the most critical and rate limiting enzyme during melanin biosynthesis. In order to develop new tyrosinase inhibitor we have synthesized 14 diphenyl ether compounds possessing hydroxyl, bromo, and nitro groups in the structure. Among the compounds prepared, 18 and 19 have shown much stronger inhibition of tyrosinase monophenolase function than arbutin used as a positive control. Both compounds 18 and 19 possess para-hydroxyphenyl moiety in their structure, which might reinforce the importance of $p$-hydroxyphenyl group in the tyrosinase inhibitory process. In the DPPH radical scavenging activity test, none of the compounds even 18 and 19 showed significant antioxidant activity. The results suggest that elaborate adjustment of diphenyl ether analogues with proper substituents have potential to be developed as new skin whitening agents working on the tyrosinase function.
\end{abstract}

Key Words: Tyrosinase inhibitor, Hydroxynitrodiphenyl ether, DPPH, Melanogenesis, Skin whitening

\section{Introduction}

Tyrosinase also known as polyphenol oxidase (PPO) is a multifunctional copper containing glycoprotein which induces the browning of fruits, vegetables and mammalian melanogenesis. Since the discovery of tyrosinase by Bourquelot and Bertrand in 1895 from the mushroom Russula nigricans, a number of studies have been made on targeting tyrosianse. ${ }^{1,2}$ Tyrosinase ubiquitously existing from microorganisms to animals and plants is known to be the most critical and rate limiting enzyme during melanin biosynthesis. ${ }^{3}$ It catalyzes first two steps in the melanin biosynthesis, the ortho-hydroxylation of tyrosine (monophenol type) to DOPA (o-diphenol type) and oxidation of DOPA to dopaquinone (o-quinone type). This resulting quinone can be subsequently transformed into melanin pigments through a series of enzymatic and non-enzymatic reactions. ${ }^{4-6}$ The inhibition of tyrosinase is most common approach to achieve skin depigmentation as this enzyme catalyzes the rate limiting steps of the melanin biosynthesis. ${ }^{7}$

To date numbers of tyrosinase inhibitors are developed from synthesis and natural resources. Most of them are phenol/catechol derivatives, structurally similar to tyrosine or DOPA, which acts as alternative substrates of tyrosinase. ${ }^{8}$ Tyrosinase inhibitors are often constructed from one of two distinct substructures such as 4-substituted resorcinol moiety and catechols. ${ }^{9}$ Depending on their chemical structures, compounds with electron donor groups in their molecule may act as substrates and those with powerful electron acceptor groups may acts as competitive inhibitors of tyrosinase. ${ }^{10}$ The 4 -substituted resorcinol group has ability to bind into binuclear active site of the enzyme and inhibit its function. Catechol structure, with two $\mathrm{OH}$ groups at $o$-positions, may behave as a chelator with the copper ions in the tyrosinase. ${ }^{11}$

Kojic acid ${ }^{12}$ and $\operatorname{arbutin}^{13}$ are widely known hypopigmenting agents (Figure 1). Recently, bibenzyl analogues are reported to have potent anti-tyrosinase activity with almost 20 -fold strength than kojic acid. ${ }^{14}$ Polyhydroxydiphenyl ether isolated from endophytic fungus has antioxidant activity, ${ }^{15}$ which has potential to be effective on tyrosinase function.

Hydroxydiphenyl ethers are useful intermediates in organic synthesis and are found in a large number of biologically active compounds. ${ }^{16}$ Many reports, recently, have demonstrated that the diphenyl ether scaffolds are found in a number of natural products and biologically important molecules. These compounds have shown diverse pharmacological activities, such as antimitotic, immunosuppressive and antibiotic activity, and are used in the control of weeds. ${ }^{17}$ Diarylbromophenols isolated from marine red alga showed potent DPPH scavenging activity. Free radicals attack biological molecules leading to many diseases associated with cell injury. ${ }^{18}$ Although diverse biological spectrum of hydroxydiphenyl ethers has been reported, little attention has been paid to the anti-tyrosinase activity of the<smiles>O=c1cc(CO)occ1O</smiles>

Kojic acid

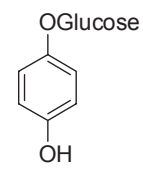

Arbutin

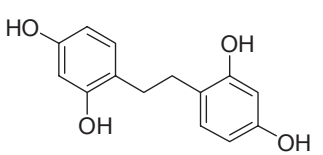

Bibenzyl analogues

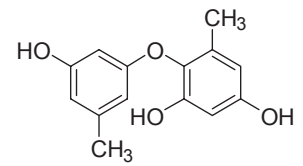

Polyhydroxydiphenylether

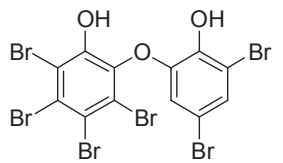

Polybromohydroxyphenyl ether
Figure 1. Tyrosinase inhibitors and biological active components from marine resources. 


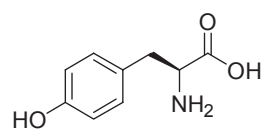

L-Tyrosine

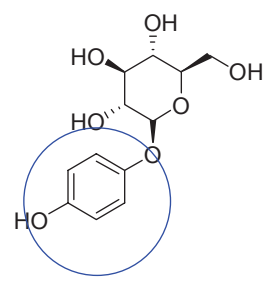

Arbutin

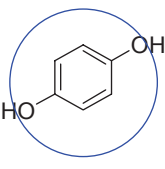

Hydroquinone

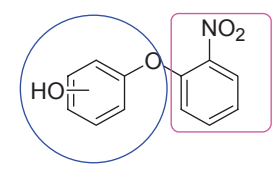

Hydroxydiphenyl ether

Figure 2. Structural similarity of hydroxynitrodiphenyl ether with L-tyrosine, arbutin, and hydroquinone.

compounds. In an effort to find potent anti-tyrosinase agent, we carried out our study to find a lead compound with hydroxyphenyl ether nucleus. For this purpose we considered the parahydroxyphenyl part of L-tyrosine, arbutin and hydroquinone as common structure. Finally, we chose nitrophenyl as a one aryl ether part in the structure, which controls the electrostatic property of the diphenyl ether (Figure 2).

\section{Experimental Section}

General. Most of the chemicals and reagents used were obtained from Aldrich Chemical Co. and some were from other companies like Junsei, Acros Organics, Tokyo Chemicals. Melting points were measured without correction in open capillaries with Barnstead Electrothermal melting point apparatus, Manual MEL-TEMP (Model No: 1202D). Chromatographic separations were monitored by thin-layer chromatography (TLC) using commercially available pre-coated Merck Kieselgel $60 \mathrm{~F}_{254}$ plate $(0.25 \mathrm{~mm})$ and detected by visualizing under UV at 254 and $365 \mathrm{~nm}$. Silica gel column chromatography (SGC) was carried out with Merck Kieselgel 60 (0.040 - 0.063 mm). All solvents used for chromatography were directly used without distillation. NMR spectra were recorded on Varian AS 400 $\left({ }^{1} \mathrm{H} \mathrm{NMR}\right.$ at $400 \mathrm{MHz}$ and ${ }^{13} \mathrm{C}$ NMR at $100 \mathrm{MHz}$ ) with tetramethylsilane (TMS) as an internal standard. Chemical shift $(\delta)$ values are expressed in ppm and coupling constant $(J)$ values in hertz (Hz). GC mass data were recorded using Agilent-5975C MSD (U.S.A) and Shimadzu GCMS-QP2010 (Japan).

General procedure A. To the round bottom flask containing proper phenol compound (1.0 eq.), were added sodium hydride (1.6 eq.) and copper (I) chloride (3.0 eq.). $N, N$-dimethylformamide $(10-20 \mathrm{~mL})$ was injected under nitrogen at room temperature followed by $5 \mathrm{~min}$. stirring. After the addition of bromonitrobenzene, the reaction mixture was refluxed for $3 \mathrm{~h}$ under nitrogen condition. Reaction mixture was cooled to rt and diluted with ethyl acetate (EtOAc). The organic layer was washed with $1.0 \mathrm{M} \mathrm{HCl}$, water, brine and dried with anhydrous $\mathrm{Na}_{2} \mathrm{SO}_{4}$. Solvent was removed under reduced pressure and purified by silica gel column chromatography to yield the product.

General procedure B. To the solution of diphenyl ether intermediate in 1,2-dichloroethane was added dropwise boron tribromide $\left(\mathrm{BBr}_{3}\right)$ solution under nitrogen atmosphere at $\mathrm{rt}$ and stirred for $30 \mathrm{~h}$ at rt. After addition of diethyl ether, the reaction mixture was further stirred for $15 \mathrm{~min}$ at $\mathrm{rt}$. Ethyl acetate was added to the mixture and the organic layer was washed with $\mathrm{H}_{2} \mathrm{O}$ and brine successively, and dried over anhydrous $\mathrm{Na}_{2} \mathrm{SO}_{4}$. Solvent was removed under reduced pressure and residue was purified.

2-Hydroxy-2'-nitrodiphenyl ether (3): Following the general procedure B (rt, $30 \mathrm{~h})$, compound 1 (104 mg, $0.43 \mathrm{mmol}$ ) was demethylated using $\mathrm{BBr}_{3}(215 \mathrm{mg}, 0.86 \mathrm{mmol})$ in 1,2-dichloroethane $(3 \mathrm{~mL})$. Purification by silica gel column chromatography (EtOAc : $n$-hexane $=1: 8$ ) afforded compound 3 (21 mg, $46.3 \%)$ as an oil. $R_{f}=0.42($ EtOAc $: n$-hexane $=1: 3) ;{ }^{1} \mathrm{H}$ NMR $\left(400 \mathrm{MHz}, \mathrm{CDCl}_{3}\right) \delta 6.90(\mathrm{ddd}, 1 \mathrm{H}, J=1.6,7.6,8.4 \mathrm{~Hz}$, H-5), 7.05 (dd, $1 \mathrm{H}, J=1.2,8.0 \mathrm{~Hz}, \mathrm{H}-3$ ), 7.08 (dd, 2H, $J=1.6$, $8.0 \mathrm{~Hz}, \mathrm{H}-6,6$ ') 7.15 (ddd, 1H, J=1.6, 7.6, 8.4 Hz, H-4), 7.20 (td, $\left.1 \mathrm{H}, J=1.2,8.4 \mathrm{~Hz}, \mathrm{H}-4^{\prime}\right), 7.50$ (ddd, $1 \mathrm{H}, J=1.6,8.0,9.2$ $\mathrm{Hz}, \mathrm{H}-5$ ') 7.92 (dd, 1H, $J=1.6,8.0 \mathrm{~Hz}, \mathrm{H}-3$ ') ${ }^{13}{ }^{\mathrm{C}} \mathrm{NMR}(100$ $\left.\mathrm{MHz}, \mathrm{CDCl}_{3}\right)$ 117.6, 119.0, 120.8, 120.9, 123.6, 126.0, 126.9, 134.6, 140.9, 142.2, 148.2, 150.6 ppm; EI-MS (m/z) 231.0 [M] ${ }^{+}$.

4'-Bromo-2-hydroxy-2'-nitrodiphenyl ether (4): Following the general procedure $\mathrm{B}(\mathrm{rt}, 30 \mathrm{~h})$, compound 2 (151 mg, 0.47 mmol) was demethylated using $\mathrm{BBr}_{3}(240 \mathrm{mg}, 0.94 \mathrm{mmol})$ in 1,2-dichloroethane ( $3 \mathrm{~mL})$. Purification by silica gel column chromatography (EtOAc : $n$-hexane $=1: 8)$ compound 4 (48 $\mathrm{mg}, 33.0 \%)$ as a gray semi-solid. $R_{f}=0.46($ EtOAc $: n$-hexane $=$ $1: 3) ;{ }^{1} \mathrm{H}$ NMR $\left(400 \mathrm{MHz}, \mathrm{CDCl}_{3}\right) \delta 6.18(\mathrm{~s}, 1 \mathrm{H}, \mathrm{OH}-2), 6.91$ (td, $1 \mathrm{H}, J=1.2,8.8 \mathrm{~Hz}, \mathrm{H}-5), 7.01$ (d, 2H, $J=9.2 \mathrm{~Hz}, \mathrm{H}-3,6)$, 7.07 (dd, 1H, $J=1.6,8.4$ Hz, H-6'), 7.15 (ddd, $1 \mathrm{H}, J=1.2$, 8.4, 9.2 Hz, H-4), 7.45 (dd, 1H, J=1.6, 9.2 Hz, H-5'), 7.91 (d, $1 \mathrm{H}, J=2.8, \mathrm{~Hz}, \mathrm{H}-3$ '); ${ }^{13} \mathrm{C} \mathrm{NMR}\left(100 \mathrm{MHz}, \mathrm{CDCl}_{3}\right) 117.8$, $120.2,120.8,121.1,125.9,127.2,128.7,134.6,140.8,142.0$, 148.0, 149.4 ppm; EI-MS $(\mathrm{m} / \mathrm{z}) 265.0\left[\mathrm{M}+\mathrm{H}-\mathrm{NO}_{2}\right]^{+}$.

3-Methoxy-2'-nitrodiphenyl ether (5): Following the general procedure A, guaiacol (1.0 g, $8.06 \mathrm{mmol})$ was coupled with 2-bromo-nitrobenzene (1.63 g, $8.06 \mathrm{mmol})$ using sodium hydride $(0.320 \mathrm{~g}, 13.30 \mathrm{mmol})$ and copper (I) chloride (2.43 g, 24.62 $\mathrm{mmol}$ ) in DMF. The crude product was purified by silica gel column chromatography (EtOAc $: n$-hexane $=1: 8$ ) to yield compound $\mathbf{5}(341 \mathrm{mg}, 17.3 \%)$ as a light yellow crystalline solid. $R_{f}=0.51($ EtOAc $: n$-hexane $=1: 3) ; \operatorname{mp~} 54-56{ }^{\circ} \mathrm{C} ;{ }^{1} \mathrm{H} \mathrm{NMR}$ $\left(400 \mathrm{MHz}, \mathrm{CDCl}_{3}\right) \delta 3.79$ (s, $\left.3 \mathrm{H}, \mathrm{OCH}_{3}-3\right), 6.61$ (dd, $1 \mathrm{H}, J=2.4$, $4.0 \mathrm{~Hz}, \mathrm{H}-2), 6.62$ (d, 1H, $J=8.8 \mathrm{~Hz}, \mathrm{H}-6), 6.73$ (dd, $1 \mathrm{H}, J=$ 1.6, 8.4 Hz, H-4), 7.05 (dd, 1H, $J=0.8,8.4$ Hz, H-6'), 7.20 (td, $1 \mathrm{H}, J=1.2,8.4 \mathrm{~Hz}, \mathrm{H}-5), 7.26$ (t, $1 \mathrm{H}, J=8.4 \mathrm{~Hz}, \mathrm{H}-4$ ') 7.50 (ddd, $1 \mathrm{H}, J=1.6,8.4,8.8 \mathrm{~Hz}, \mathrm{H}-5$ '), 7.95 (dd, 1H, $J=1.6,8.0 \mathrm{~Hz}$, H-3'); ${ }^{13} \mathrm{C}$ NMR (100 MHz, $\left.\mathrm{CDCl}_{3}\right)$ 55.7, 105.5, 110.5, 111.4, $120.9,123.5,125.9,130.7,134.3,141.5,150.7,157.1,161.3$ ppm; EI-MS $(\mathrm{m} / \mathrm{z}) 245.0[\mathrm{M}]^{+}$. 
3-Hydroxy-2'-nitrodiphenyl ether (6), 6-bromo-3-hydroxy2'-nitrodiphenyl ether (7) and 4-bromo-3-hydroxy-2'-nitrodiphenyl ether (8): Following the general procedure B (rt, $27 \mathrm{~h})$, compound 5 (78 mg, $0.32 \mathrm{mmol}$ ) in 1,2-dichloroethane (4 mL), was demethylated using $\mathrm{BBr}_{3}(160 \mathrm{mg}, 0.64 \mathrm{mmol})$. Purification by silica gel column chromatography $($ EtOAc $: n$-hexane $=$ $1: 7)$ afforded compounds 6 (43 $\mathrm{mg}, 58.1 \%)$ as a gray semisolid, 7 (11 mg, 11.1\%) as a dark brown solid and $8(7 \mathrm{mg}$, $7.0 \%$ ) as a viscous liquid. Compound 6: $R_{f}=0.34$ (EtOAc : $n$-hexane $=1: 3) ;{ }^{1} \mathrm{H}$ NMR $\left(400 \mathrm{MHz}, \mathrm{CDCl}_{3}\right) \delta 5.23$ (brs, $1 \mathrm{H}$, $\mathrm{OH}-3), 6.53$ (t, $1 \mathrm{H}, J=2.0 \mathrm{~Hz}, \mathrm{H}-2), 6.59$ (dd, $1 \mathrm{H}, J=2.0,8.4$ $\mathrm{Hz}, \mathrm{H}-4), 6.64$ (dd, 1H, $J=2.4,8.4 \mathrm{~Hz}, \mathrm{H}-6), 7.06$ (d, 1H, $J=$ 8.4 Hz, H-6'), 7.20 (t, 2H, J=8.4 Hz, H-5, 4'), 7.51 (td, 1H, $J=1.6,8.4 \mathrm{~Hz}, \mathrm{H}-5$ '), 7.94 (dd, $1 \mathrm{H}, J=1.6,8.0 \mathrm{~Hz}, \mathrm{H}-3$ ') ${ }^{13}{ }^{\mathrm{C}}$ NMR $\left(100 \mathrm{MHz}, \mathrm{CDCl}_{3}\right)$ 106.8, 111.4, 111.8, 121.2, 123.7, 125.9, 131.0, 134.5, 141.6, 150.54, 157.2, 157.3 ppm; EI-MS $(\mathrm{m} / \mathrm{z}) 231.1[\mathrm{M}]^{+}$. Compound 7: $R_{f}=0.35($ EtOAc $: n$-hexane $=$ $1: 3)$; mp $82-84{ }^{\circ} \mathrm{C} ;{ }^{1} \mathrm{H} \mathrm{NMR}\left(400 \mathrm{MHz}, \mathrm{CDCl}_{3}\right) \delta 5.64$ (brs, $1 \mathrm{H}, \mathrm{OH}-3), 6.53$ (dd, $1 \mathrm{H}, J=2.4,8.0 \mathrm{~Hz}, \mathrm{H}-4), 6.69$ (d, $1 \mathrm{H}, J=$ $2.8 \mathrm{~Hz}, \mathrm{H}-2), 7.09$ (d, 1H, $J=8.4 \mathrm{~Hz}, \mathrm{H}-6$ '), 7.26 (t, 1H, $J=$ $7.2 \mathrm{~Hz}, \mathrm{H}-4$ ') 7.42 (d, 1H, $J=8.8 \mathrm{~Hz}, \mathrm{H}-5), 7.55$ (td, $1 \mathrm{H}, J=$ $1.2,8.4 \mathrm{~Hz}, \mathrm{H}-5^{\prime}$ ), 7.97 (dd, $\left.1 \mathrm{H}, J=2.8,8.0 \mathrm{~Hz}, \mathrm{H}-3{ }^{\prime}\right) ;{ }^{\prime 3} \mathrm{C}$ NMR (100 MHz, $\left.\mathrm{CDCl}_{3}\right)$ 105.2, 106.8, 112.4, 121.8, 124.4, 126.1, 132.9, 134.6, 141.9, 149.8, 153.6, 157.2 ppm; EI-MS $(\mathrm{m} / \mathrm{z}) 308.9[\mathrm{M}]^{+}, 310.9[\mathrm{M}+2]^{+}$; Relative peak intensity $=1: 1$. Compound 8: $R_{f}=0.21($ EtOAc $: n$-hexane $=1: 3) ;{ }^{1} \mathrm{H}$ NMR $\left(400 \mathrm{MHz}, \mathrm{CDCl}_{3}\right) \delta 5.44$ (brs, $\left.1 \mathrm{H}, \mathrm{OH}-3\right), 6.53$ (d, $1 \mathrm{H}, J=2.8$ Hz, H-2), 6.60 (dd, 1H, $J=2.8,8.4 \mathrm{~Hz}, \mathrm{H}-6), 6.90$ (dd, 1H, $J$ $=1.6,8.0 \mathrm{~Hz}, \mathrm{H}-6$ ') 7.21 (ddd, $1 \mathrm{H}, J=1.2,7.2,8.4 \mathrm{~Hz}, \mathrm{H}-4$ '), $7.45(\mathrm{~d}, 1 \mathrm{H}, J=8.4 \mathrm{~Hz}, \mathrm{H}-5), 7.51$ (ddd, $1 \mathrm{H}, J=1.6,7.6,8.4$ Hz, H-5'), 7.98 (dd, 1H, $\left.J=1.6,8.0 \mathrm{~Hz}, \mathrm{H}-3^{\prime}\right) ;{ }^{13} \mathrm{C}$ NMR (100 $\left.\mathrm{MHz}, \mathrm{CDCl}_{3}\right)$ 105.5, 108.8, 114.0, 119.9, 123.8, 126.2, 134.6, 134.7, 140.8, 150.1, 153.1, 156.6 ppm; EI-MS $(m / z) 309.0[\mathrm{M}]^{+}$, $311.0[\mathrm{M}+2]^{+}$; Relative peak intensity $=1: 1$.

4'-Bromo-3-methoxy-2'-nitrodiphenyl ether (9): Following the general procedure A, 3-methoxy phenol $(0.7 \mathrm{~g}, 5.64 \mathrm{mmol})$ was coupled with 2,5-dibromonitrobenzene (1.9 g, $5.64 \mathrm{mmol})$ using sodium hydride $(0.24 \mathrm{~g}, 9.31 \mathrm{mmol})$ and copper (I) chloride $(1.7 \mathrm{~g}, 17.24 \mathrm{mmol})$ in DMF $(15 \mathrm{~mL})$. The crude product was purified by silica gel column chromatography (EtOAc : $n$-hexane $=1: 10)$ to yield compound $9(665 \mathrm{mg}, 36.34 \%)$ as an orange colored crystalline solid. $R_{f}=0.27$ (EtOAc $: n$-hexane $=$ $1: 5) ; \mathrm{mp} 44-46{ }^{\circ} \mathrm{C} ;{ }^{1} \mathrm{H}$ NMR $\left(400 \mathrm{MHz}, \mathrm{CDCl}_{3}\right) \delta 3.79$ (s, $\left.3 \mathrm{H}, \mathrm{OCH}_{3}-3\right), 6.59$ (dd, $\left.1 \mathrm{H}, J=2.4,2.4 \mathrm{~Hz}, \mathrm{H}-2\right), 6.60$ (dd, $1 \mathrm{H}, J=\overline{2} .4,2.4 \mathrm{~Hz}, \mathrm{H}-6), 6.75$ (dt, $1 \mathrm{H}, J=1.2,8.4 \mathrm{~Hz}, \mathrm{H}-4)$, 7.00 (d, 1H, $J=8.8 \mathrm{~Hz}, \mathrm{H}-6$ ') 7.27 (t, 1H, $J=8.8 \mathrm{~Hz}, \mathrm{H}-5$ ), 7.46 (dd, $\left.1 \mathrm{H}, J=2.8,8.8 \mathrm{~Hz}, \mathrm{H}-5^{\prime}\right), 7.95(\mathrm{~d}, 1 \mathrm{H}, J=2.8 \mathrm{~Hz}$, H-3'); ${ }^{13} \mathrm{C} \mathrm{NMR}\left(100 \mathrm{MHz}, \mathrm{CDCl}_{3}\right)$ 55.7, 105.6, 110.8, 111.3, 122.0, 125.8, 128.5, 130.8, 134.3, 140.8, 149.5, 156.7, 161.4 ppm; EI-MS $(\mathrm{m} / \mathrm{z}) 323.0[\mathrm{M}]^{+}, 325.0[\mathrm{M}+2]^{+}$; Relative peak intensity $=1: 1$.

4',6-Dibromo-3-hydroxy-2'-nitrodiphenyl ether (10) and 4'-bromo-3-hydroxy-2'-nitrodiphenyl ether (11): Following the general procedure B ( $\mathrm{rt}, 28 \mathrm{~h}$ ), compound 9 (134 mg, 0.43 $\mathrm{mmol}$ ) in 1, 2-dichloroethane (4 mL), was demethylated using $\mathrm{BBr}_{3}(220 \mathrm{mg}, 0.87 \mathrm{mmol})$. Purification by silica gel column chromatography (EtOAc : $n$-hexane $=1: 10$ ) afforded compound $\mathbf{1 0}(25 \mathrm{mg}, 15.0 \%)$ as a greenish yellow crystalline solid and compound $\mathbf{1 1}$ (58 mg, 44.2\%) as a dark brown oil. Compound 10: $R_{f}=0.33($ EtOAc $: n$-hexane $=1: 3)$; mp $72-\overline{74{ }^{\circ} \mathrm{C}}$; ${ }^{\mathrm{I}} \mathrm{H} \mathrm{NMR}\left(400 \mathrm{MHz}, \mathrm{CDCl}_{3}\right) \delta 5.63$ (brs, $\left.1 \mathrm{H}, \mathrm{OH}-3\right), 6.52$ (dd, $1 \mathrm{H}, J=2.4,8.8 \mathrm{~Hz}, \mathrm{H}-4), 6.69$ (d, 1H, $J=2.4 \overline{\mathrm{Hz}}, \mathrm{H}-2), 7.04$ (d, $1 \mathrm{H}, J=9.2 \mathrm{~Hz}, \mathrm{H}-6$ ') 7.44 (d, 1H, $J=8.4 \mathrm{~Hz}, \mathrm{H}-5), 7.51$ (dd, $1 \mathrm{H}, J=2.0,8.8 \mathrm{~Hz}, \mathrm{H}-5$ ') 7.96 (d, $1 \mathrm{H}, J=1.6 \mathrm{~Hz}, \mathrm{H}-3^{\prime}$ ); ${ }^{13} \mathrm{C}$ NMR (100 MHz, $\left.\mathrm{CDCl}_{3}\right)$ 105.6, 106.9, 112.4, 122.9, 126.0, 129.4, 133.0, 134.6, 141.8, 148.6, 153.7, 156.8 ppm; EI-MS $(\mathrm{m} / \mathrm{z}) 342.9\left[\mathrm{M}-\mathrm{NO}_{2}\right]^{+}$. Compound 11: $R_{f}=0.30($ EtOAc : $n$-hexane $=1: 3) ;{ }^{1} \mathrm{H} \mathrm{NMR}\left(400 \mathrm{MHz}, \mathrm{CDCl}_{3}\right) \delta 5.24$ (brs, $1 \mathrm{H}$, $\mathrm{OH}-3), 6.53$ (t, $1 \mathrm{H}, J=2.4 \mathrm{~Hz}, \mathrm{H}-2), 6.58$ (dd, $1 \mathrm{H}, J=2.4,8.0$ $\mathrm{Hz}, \mathrm{H}-4), 6.66$ (dd, 1H, $J=2.4,8.0 \mathrm{~Hz}, \mathrm{H}-6), 7.01$ (d, 1H, $J=8.8$ Hz, H-6'), 7.21 (t, 1H, $J=8.0$ Hz, H-5), 7.47 (dd, 1H, $J=2.8,8.8$ $\mathrm{Hz}, \mathrm{H}-5$ ') 7.94 (d, $\left.1 \mathrm{H}, J=2.8 \mathrm{~Hz}, \mathrm{H}-3^{\prime}\right)$; ${ }^{13} \mathrm{C}$ NMR (100 MHz, $\left.\mathrm{CDCl}_{3}\right)$ 106.8, 111.4, 112.2, 122.2, 125.8, 128.7, 131.1, 134.5, 141.5, 149.3, 156.8, 157.3 ppm; EI-MS $(\mathrm{m} / \mathrm{z}) 309.0[\mathrm{M}]^{+}, 311.0$ $[\mathrm{M}+2]^{+}$; Relative peak intensity $=1: 1$.

3,5-Dimethoxy-2'-nitrodiphenyl ether (12): Following the general procedure A, 3-methoxy phenol ( $0.7 \mathrm{~g}, 4.54 \mathrm{mmol})$ was coupled with 2-bromo-nitrobenzene ( $0.91 \mathrm{~g}, 4.54 \mathrm{mmol})$ using sodium hydride $(0.18 \mathrm{~g}, 7.50 \mathrm{mmol})$ and copper (I) chloride $(1.37 \mathrm{~g}, 13.87 \mathrm{mmol})$ in DMF $(15 \mathrm{~mL})$. The crude product was purified by silica gel column chromatography (EtOAc : $n$ hexane $=1: 8)$ to provide compound $12(195 \mathrm{mg}, 11.68 \%)$ as a brown colored oil. $R_{f}=0.31($ EtOAc $: n$-hexane $=1: 5) ;{ }^{1} \mathrm{H}$ NMR (400 MHz, $\mathrm{CDCl}_{3}$ ) $\delta 3.74\left(\mathrm{~s}, 6 \mathrm{H}, \mathrm{OCH}_{3}-3,5\right), 6.18$ (d, $2 \mathrm{H}, J=2.0 \mathrm{~Hz}, \mathrm{H}-2,6), 6.26$ (t, $1 \mathrm{H}, J=2.0 \mathrm{~Hz}, \mathrm{H}-4), 7.07$ (dd, $1 \mathrm{H}, J=1.2,8.4 \mathrm{~Hz}, \mathrm{H}-6$ '), 7.18 (ddd, $1 \mathrm{H}, J=1.2,7.6,8.0 \mathrm{~Hz}$, H-4'), 7.49 (ddd, $1 \mathrm{H}, J=1.2,7.2,8.8 \mathrm{~Hz}, \mathrm{H}-5$ '), 7.93 (dd, $1 \mathrm{H}$, $J=1.6,8.4 \mathrm{~Hz}, \mathrm{H}-3$ '); ${ }^{13} \mathrm{C} \mathrm{NMR}\left(100 \mathrm{MHz}, \mathrm{CDCl}_{3}\right) 55.7$, $96.9,97.9,121.2,123.6,125.9,134.4,141.6,150.5,157.8$, 162.0 ppm; EI-MS $(\mathrm{m} / \mathrm{z}) 275.1[\mathrm{M}]^{+}$.

3,5-Dihydroxy-2'-nitrodiphenyl ether (13), 4-bromo-3,5dihydroxy-2'-nitrodiphenyl ether (14) and 6-bromo-3,5-dihydroxy-2' -nitrodiphenyl ether (15): Following the general procedure $\mathrm{B}$ ( $\mathrm{rt}, 14 \mathrm{~h})$, compound 12 (103 $\mathrm{mg}, 0.37 \mathrm{mmol}$ ) was demethylated using $\mathrm{BBr}_{3}$ (94 mg, $0.37 \mathrm{mmol}$ ) in 1,2-dichloroethane $(4 \mathrm{~mL})$. Purification by silica gel column chromatography (EtOAc $: n$-hexane $=1: 4)$ afforded compound 13 (12 $\mathrm{mg}, 13.2 \%)$ as a greenish brown solid, compound $14(10 \mathrm{mg}$, $8.2 \%)$ as a dark brown solid and compound $\mathbf{1 5}(22 \mathrm{mg}, 18.1 \%)$ as an oil. Compound 13: $R_{f}=0.44($ EtOAc $: n$-hexane $=1: 1)$; mp 154-156 ${ }^{\circ} \mathrm{C} ;{ }^{1} \mathrm{H} \mathrm{NMR}\left(400 \mathrm{MHz}, \mathrm{CDCl}_{3} / \mathrm{CD}_{3} \mathrm{OD}\right) \delta 6.05$ $(\mathrm{d}, 2 \mathrm{H}, J=2.0 \mathrm{~Hz}, \mathrm{H}-2,6), 6.14$ (t, $1 \mathrm{H}, J=2.0 \mathrm{~Hz}, \mathrm{H}-4), 7.09$ (dd, $1 \mathrm{H}, J=1.2,8.4 \mathrm{~Hz}, \mathrm{H}-6$ '), 7.17 (td, $1 \mathrm{H}, J=1.2,8.0 \mathrm{~Hz}$, H-4'), 7.50 (ddd, $1 \mathrm{H}, J=1.6,7.6,8.8 \mathrm{~Hz}, \mathrm{H}-5$ '), 7.92 (dd, 1H, $\left.J=2.0,8.0 \mathrm{~Hz}, \mathrm{H}-3{ }^{\prime}\right) ;{ }^{13} \mathrm{C}$ NMR (100 MHz, $\left.\mathrm{CDCl}_{3} / \mathrm{CD}_{3} \mathrm{OD}\right)$ 98.6, 99.3, 121.1, 123.2, 125.7, 134.4, 141.3, 150.7, 157.6, 159.0 ppm; EI-MS $(\mathrm{m} / \mathrm{z}) 247.0[\mathrm{M}]^{+}$. Compound 14: $R_{f}=0.50$ (EtOAc : $n$-hexane $=1: 1) ; \mathrm{mp} 82-84{ }^{\circ} \mathrm{C} ;{ }^{1} \mathrm{H} \mathrm{NMR}(400 \mathrm{MHz}$, $\left.\mathrm{CDCl}_{3}\right) \delta 5.58$ (brs, 2H, OH-3, 5), 6.29 (s, 2H, H-2, 6), 7.13 (dd, $1 \mathrm{H}, J=1.2,8.4 \mathrm{~Hz}, \mathrm{H}^{-6}$ '), 7.28 (td, $1 \mathrm{H}, J=1.2,7.6 \mathrm{~Hz}$, H-4'), 7.57 (ddd, $1 \mathrm{H}, J=2.8,8.8,9.6 \mathrm{~Hz}, \mathrm{H}-5$ ') 7.97 (dd, $1 \mathrm{H}$, $J=1.2,8.0 \mathrm{~Hz}, \mathrm{H}-3$ '); ${ }^{13} \mathrm{C} \mathrm{NMR}\left(100 \mathrm{MHz}, \mathrm{CDCl}_{3}\right) 94.4$, $98.9,122.3,124.6,126.1,134.6,142.0,149.4,154.0,157.7$ ppm; EI-MS $(\mathrm{m} / \mathrm{z}) 325.0[\mathrm{M}]^{+}, 327.0[\mathrm{M}+2]^{+}$; Relative peak intensity $=1: 1$. Compound 15: $R_{f}=0.45($ EtOAc $/ n$-Hexane,

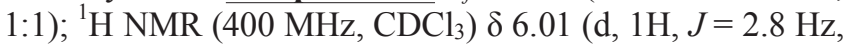


H-4), 6.39 (d, 1H, $J=2.8 \mathrm{~Hz}, \mathrm{H}-2), 6.99$ (dd, 1H, $J=1.2,8.4$ Hz, H-6'), 7.23 (td, 1H, J=1.2, 8.4 Hz, H-4'), 7.53 (ddd, 1H, $J=1.6,7.2,8.4$ Hz, H-5'), 7.98 (dd, 1H, $J=1.6,8.0$ Hz, H-3'); ${ }^{13} \mathrm{C} \mathrm{NMR}\left(100 \mathrm{MHz}, \mathrm{CDCl}_{3}\right)$ 94.3, 100.0, 100.5, 120.6, 124.2, 126.2, 134.7, 141.0, 149.7, 153.7, 154.8, 157.0 ppm; EI-MS $(\mathrm{m} / \mathrm{z}) 325.0[\mathrm{M}]^{+}, 327.0[\mathrm{M}+2]^{+}$; Relative peak intensity $=1: 1$.

4'-Bromo-4-methoxy-2'-nitrodiphenyl ether (16): Following the general procedure A, 4-methoxyphenol $(1.2 \mathrm{~g}, 9.67 \mathrm{mmol})$ was coupled with 2,5-dibromonitrobenzene (2.72 g, $9.67 \mathrm{mmol})$ using sodium hydride $(0.38 \mathrm{~g}, 15.97 \mathrm{mmol})$ and copper (I) chloride $(2.92 \mathrm{~g}, 29.55 \mathrm{mmol})$ in DMF $(20 \mathrm{~mL})$. The crude product was purified by silica gel column chromatography (EtOAc : $n$-hexane $=1: 8)$ to yield compound $\mathbf{1 6}(475 \mathrm{mg}, 15.2 \%)$ as an orange colored semisolid. $R_{f}=0.57$ (EtOAc $: n$-hexane $=1$ : 3); ${ }^{1} \mathrm{H}$ NMR (400 MHz, $\left.\mathrm{CDCl}_{3}\right) \delta 3.81\left(\mathrm{~s}, 3 \mathrm{H}, \mathrm{OCH}_{3}-4\right), 6.87$, $6.91(\mathrm{~d}, 2 \mathrm{H}, J=9.2 \mathrm{~Hz}, \mathrm{H}-3,5), 7.0(\mathrm{~d}, 2 \mathrm{H}, J=9.2 \overline{\mathrm{Hz}}, \mathrm{H}-2,6)$, 7.23 (d, 1H, $J=8.4$ Hz, H-6'), 7.40, 7.68 (dd, 1H, $J=2.0,8.4$ Hz, H- 5'), 7.92, 8.23 (d, 1H, J=2.0 Hz, H-3'); ${ }^{13} \mathrm{C}$ NMR (100 $\left.\mathrm{MHz}, \mathrm{CDCl}_{3}\right)$ 55.9, 115.5, 121.3, 125.4, 131.7, 133.9, 135.7, 140.8, 148.4, 150.9, 157.2 ppm; EI-MS $(\mathrm{m} / \mathrm{z}) 323.0[\mathrm{M}]^{+}$; $325.0[\mathrm{M}+2]^{+}$; Relative peak intensity $=1: 1$.

4-Methoxy-2'-nitrodiphenyl ether(17): Following the general procedure A, 4-methoxyphenol (3.00 g, $24.16 \mathrm{mmol})$ was coupled with 1-bromo-2-nitrobenzene (4.88 g, $24.16 \mathrm{mmol})$ using sodium hydride $(0.96 \mathrm{~g}, 39.92 \mathrm{mmol})$ and copper (I) chloride $(7.31 \mathrm{~g}, 73.84 \mathrm{mmol})$ in DMF $(30 \mathrm{~mL})$. The crude product was purified by silica gel column chromatography (EtOAc : $n$-hexane $=1: 8)$ to yield compound $17(2.45 \mathrm{~g}, 41.35 \%)$ as a light yellow solid. $R_{f}=0.51($ EtOAc $: n$-hexane $=1: 3) ; \mathrm{mp}$ 74-76 ${ }^{0} \mathrm{C} ;{ }^{1} \mathrm{H} \mathrm{NMR}\left(400 \mathrm{MHz}, \mathrm{CDCl}_{3}\right) \delta 3.81$ (s, 3H, $\left.\mathrm{OCH}_{3}-4\right)$, 6.89-6.93 (m, 3H, H-3, 5, 6') 7.01 (dd, 2H, $J=2.8,9 . \overline{2} \mathrm{~Hz}$, H-2, 6), 7.12 (ddd, $1 \mathrm{H}, J=1.2,7.6,8.4$ Hz, H-4'), 7.44 (ddd, $\left.1 \mathrm{H}, J=1.6,7.6,8.4 \mathrm{~Hz}, \mathrm{H}-5^{\prime}\right), 7.91$ (dd, $1 \mathrm{H}, J=1.6,8.0 \mathrm{~Hz}$, H-3'); ${ }^{13} \mathrm{C} \mathrm{NMR}\left(100 \mathrm{MHz}, \mathrm{CDCl}_{3}\right)$ 55.9, 115.3, 119.1, 121.3, $122.4,125.9,134.2,140.9,148.8,152.2,157.0$ ppm; EI-MS $(\mathrm{m} / \mathrm{z}) 245.0[\mathrm{M}]^{+}$

4'-Bromo-4-hydroxy-2'-nitrodiphenyl ether (18): Following the general procedure B (rt, $14 \mathrm{~h})$, compound $\mathbf{1 6}$ (120 mg, 0.37 $\mathrm{mmol})$ in 1,2-dichloroethane (3 $\mathrm{mL})$, was demethylated using $\mathrm{BBr}_{3}(140 \mathrm{mg}, 0.55 \mathrm{mmol})$. Purification by silica gel column chromatography (EtOAc : $n$-hexane $=1: 5$ ) provided compound 18 (48 $\mathrm{mg}, 42.0 \%)$ as a brown solid. $R_{f}=0.28$ (EtOAc $: n$-hexane $=1: 3) ; \operatorname{mp} 94-96{ }^{\circ} \mathrm{C} ;{ }^{1} \mathrm{H} \mathrm{NMR}(400 \mathrm{MHz}$, $\left.\mathrm{CDCl}_{3} / \mathrm{CD}_{3} \mathrm{OD}\right) \delta 6.82(\mathrm{dd}, 2 \mathrm{H}, J=2.8,8.8 \mathrm{~Hz}, \mathrm{H}-3,5), 6.84$ (d, $1 \mathrm{H}, J=8.0 \mathrm{~Hz}, \mathrm{H}-6$ '), 6.89 (dd, $2 \mathrm{H}, J=2.8,8.8 \mathrm{~Hz}, \mathrm{H}-2,6)$, 7.37 (dd, 1H, $J=2.0,9.2 \mathrm{~Hz}, \mathrm{H}-5^{\prime}$ ), 7.87 (d, $1 \mathrm{H}, J=2.4 \mathrm{~Hz}$, $\mathrm{H}-3$ '); ${ }^{13} \mathrm{C}$ NMR (100 MHz, $\left.\mathrm{CDCl}_{3} / \mathrm{CD}_{3} \mathrm{OD}\right) 116.9,120.0$, 121.5, 125.6, 127.1, 134.3, 140.5, 147.6, 151.2, 154.4 ppm; EI-MS $(m / z) 265.0\left[\mathrm{M}+\mathrm{H}-\mathrm{NO}_{2}\right]^{+}$.

4-Hydroxy-2'-nitrodiphenyl ether (19): Following the general procedure $\mathrm{B}$ (rt, $18 \mathrm{~h}$ ), compound 17 (75 mg, $0.30 \mathrm{mmol})$ was demethylated using $\mathrm{BBr}_{3}(152 \mathrm{mg}, 0.60 \mathrm{mmol})$ in 1,2dichloroethane $(3 \mathrm{~mL})$. Purification by silica gel column chromatography (EtOAc : $n$-hexane $=1: 5$ ) yielded compound 19 (58 $\mathrm{mg}, 82.25 \%)$ as a dark green solid. $R_{f} 0.28$ (EtOAc : $n$-hexane $=1: 3) ; \mathrm{mp} 102-104{ }^{\circ} \mathrm{C} ;{ }^{1} \mathrm{HNMR}\left(400 \mathrm{MHz}, \mathrm{CDCl}_{3}\right)$ $\delta 5.59$ (brs, $1 \mathrm{H}, \mathrm{OH}-1), 6.86$ (dd, $2 \mathrm{H}, J=2.8,8.8 \mathrm{~Hz}, \mathrm{H}-3,5)$, $6.92(\mathrm{dd}, 1 \mathrm{H}, J=\overline{1.2}, 8.4 \mathrm{~Hz}, \mathrm{H}-6$ '), 6.95 (dd, $2 \mathrm{H}, J=2.4,8.8$
Hz, H-2, 6), 7.13 (ddd, 1H, $J=1.2,7.6,8.4$ Hz, H-4'), 7.46 (ddd, $1 \mathrm{H}, J=1.6,7.6,8.4 \mathrm{~Hz}, \mathrm{H}-5^{\prime}$ ), 7.92 (dd, 1H, $J=1.6,8.0$ $\mathrm{Hz}, \mathrm{H}-3$ '); ${ }^{13} \mathrm{C}$ NMR (100 MHz, $\mathrm{CDCl}_{3}$ ) 116.9, 119.1, 121.5, $122.5,125.9,134.4,140.7,148.7,152.2,153.0$ ppm; EI-MS $(\mathrm{m} / \mathrm{z}) 231.0[\mathrm{M}]^{+}$.

1-Methoxy-3,5-bis-(2-nitro-phenoxy)benzene (20) and 5Hydroxy-3-methoxy-2'-nitrodiphenyl ether (21): Following the general procedure A, 3,5-dihydroxyanisole (255 mg, 1.82 mmol) was coupled with 1-bromo-2-nitrobenzene $(920 \mathrm{mg}$, $4.55 \mathrm{mmol})$ using sodium hydride (73 mg, $3.01 \mathrm{mmol})$ and copper (I) chloride (552 mg, $5.57 \mathrm{mmol}$ ) in DMF. The crude product was purified by silica gel column chromatography (EtOAc : $n$-hexane $=1: 10)$ to yield: compound $20(35 \mathrm{mg}, 5.1 \%)$ as an amber colored solid and compound 21 (26 mg, 5.5\%) as a dark brown oil. Compound $20 R_{f}=0.28$ (EtOAc : $n$-hexane $=1: 3) ; \mathrm{mp} 120-122{ }^{\circ} \mathrm{C} ;{ }^{1} \mathrm{H} \mathrm{NMR}\left(400 \mathrm{MHz}, \mathrm{CDCl}_{3}\right) \delta 3.76$ $\left(\mathrm{s}, 3 \mathrm{H}, \mathrm{OCH}_{3}-1\right), 6.26(\mathrm{t}, 1 \mathrm{H}, J=2.0 \mathrm{~Hz}, \mathrm{H}-4), 6.38$ (d, 2H, $J$ $=2.0 \mathrm{~Hz}, \overline{\mathrm{H}}-2,6), 7.12(\mathrm{~d}, 2 \mathrm{H}, J=8.4 \mathrm{~Hz}, \mathrm{H}-6,6$ '), 7.24 (td, $2 \mathrm{H}, J=1.2,8.4 \mathrm{~Hz}, \mathrm{H}-4,4^{\prime}$ ), 7.58 (ddd, $2 \mathrm{H}, J=1.2,7.6,8.8$ Hz, H-5, 5'), 7.95 (dd, 2H, $J=1.6,8.0, \mathrm{~Hz}, \mathrm{H}-3,3$ ') ${ }^{13}{ }^{\mathrm{C}} \mathrm{NMR}$ $\left(100 \mathrm{MHz}, \mathrm{CDCl}_{3}\right)$ 55.6, 101.0, 101.8, 121.7, 124.3, 126.0, 134.6, 141.7, 149.8, 158.3, 162.3 ppm; EI-MS (m/z) 382.1 [M] . Compound 21: $R_{f}=0.20($ EtOAc $: n$-hexane $=1: 3) ;{ }^{1} \mathrm{H} \mathrm{NMR}$ $\left(400 \mathrm{MHz}, \mathrm{CDCl}_{3}\right) \delta 3.74\left(\mathrm{~s}, 3 \mathrm{H}, \mathrm{OCH}_{3}-3\right), 6.11(\mathrm{t}, 1 \mathrm{H}, J=2.0$ Hz, H-6), 6.17 (t, 1H, $J=2.0 \mathrm{~Hz}, \mathrm{H}-4), 6.22$ (t, 1H, $J=2.0 \mathrm{~Hz}$, H-2), 7.09 (d, 1H, $J=8.4$ Hz, H- 6'), 7.20 (td, 1H, $J=1.2,8.0$ Hz, H-4'), 7.51 (ddd, 1H, $J=1.6,7.6,8.4$ Hz, H-5'), 7.95 (dd, $\left.1 \mathrm{H}, J=1.6,8.0, \mathrm{~Hz}, \mathrm{H}-3{ }^{\prime}\right) ;{ }^{13} \mathrm{C} \mathrm{NMR}\left(100 \mathrm{MHz}, \mathrm{CDCl}_{3}\right) 55.8$, 97.9, 98.1, 99.2, 121.4, 123.8, 125.9, 134.5, 141.6, 150.3, 157.9, 158.0, 162.1 ppm; EI-MS $(\mathrm{m} / \mathrm{z}) 261.1[\mathrm{M}]^{+}$.

1-Hydroxy-3,5-bis-(2-nitro-phenoxy)benzene (22): Following the general procedure B ( $\mathrm{rt}, 5 \mathrm{~h})$, compound 20 (34 mg, $0.09 \mathrm{mmol})$ was demethylated using $\mathrm{BBr}_{3}(23 \mathrm{mg}, 0.09 \mathrm{mmol})$ in 1,2-dichloroethane ( $3 \mathrm{~mL})$. Purification by silica gel column chromatography (EtOAc : $n$-hexane $=1: 4)$ afforded compound $22(21 \mathrm{mg}, 63.4 \%)$ as a brown solid. $R_{f}=0.54$ (EtOAc : $n$-hexane $=1: 1) ; \mathrm{mp} 102-104{ }^{\circ} \mathrm{C} ;{ }^{1} \mathrm{H} \mathrm{NMR}\left(400 \mathrm{MHz}, \mathrm{CDCl}_{3}\right)$ $\delta 5.67$ (brs, $1 \mathrm{H}, \mathrm{OH}-1), 6.25$ (t, $1 \mathrm{H}, J=1.2 \mathrm{~Hz}, \mathrm{H}-4), 6.30$ (d, $2 \mathrm{H}, J=2.4 \mathrm{~Hz}, \mathrm{H}-\overline{2}, 6), 7.13$ (d, 2H, $J=8.0 \mathrm{~Hz}, \mathrm{H}-6,6$ '), 7.25 (t, 2H, $\left.J=8.0 \mathrm{~Hz}, \mathrm{H}-4,4^{\prime}\right), 7.56$ (ddd, $2 \mathrm{H}, J=1.2,7.6,8.4 \mathrm{~Hz}$, H-5, 5'), 7.94 (dd, 2H, J=1.2, 8.0, Hz, H-3, 3'); ${ }^{13} \mathrm{C}$ NMR $\left(100 \mathrm{MHz}, \mathrm{CDCl}_{3}\right) 101.7,102.3,122.0,124.4,126.0,134.7$, 141.7, 149.7, 158.3, 158.4 ppm; EI-MS $(\mathrm{m} / \mathrm{z}) 368.1[\mathrm{M}]^{+}$.

Mushroom tyrosinase inhibition assay in vitro. ${ }^{20}$ All samples were dissolved in DMSO in a test tube and diluted with $800 \mu \mathrm{L}$ of $0.1 \mathrm{M}$ phosphate buffer ( $\mathrm{pH}$ 6.5) $\mu \mathrm{L}$ ). To this sample solution $(50 \mu \mathrm{L}), 50 \mu \mathrm{L}$ of mushroom tyrosinase $(2000 \mathrm{U} / \mathrm{mL})$ and $100 \mu \mathrm{L}$ of $1.5 \mathrm{mM}$ L-tyrosine were also added sequentially. The assay mixture was incubated at $37{ }^{\circ} \mathrm{C}$ for $10 \mathrm{~min}$. The amount of dopachrome produced was measured against a blank solution at $490 \mathrm{~nm}$ using a spectrophotometer. Percentage inhibition of tyrosinase was calculated by using the following formula.

Tyrosinase inhibition $(10 \%)=100-\left(\frac{b-b^{\prime}}{a-a^{\prime}} \times 100\right)$ 
Where $a$ and $b$ represent absorbance of blank and sample solution respectively. Similarly, $a$ ' and $b$ ' represent absorbance in the absence of tyrosinase. Each result is the mean of three concurrent readings. Arbutin was used as a positive control.

DPPH radical scavenging assay. ${ }^{21}$ All compounds used were dissolved in ethanol (Sigma, USA). In order to determine the capacity to scavenge the stable free radical, 1,1-diphenyl-2picryl-hydrazyl (DPPH) (Sigma, USA), $50 \mu \mathrm{L}$ of each compound solution was added to each well in 96-well plate containing 50 $\mu \mathrm{L}$ of $200 \mu \mathrm{M}$ DPPH stock solution. After incubation for $30 \mathrm{~min}$ with shaking at room temperature, the absorbance was read at $517 \mathrm{~nm}$ using a microplate reader (VERSAmax, Molecular Devices). The percent DPPH scavenging activity was expressed as follow:

$$
\begin{aligned}
& \text { DPPH scavenging activity }(\%)= \\
& (A c-A b)-(A s-A b)] /(A c-A b) \times 100
\end{aligned}
$$

Where $A c$ was the absorbance of the DPPH solution without compound, $A s$ was that of the DPPH solution with compound, and $A b$ was that of the blank. At least three different experiments for each compound were performed in triplicate. Vitamin $\mathrm{C}$ was used as a positive control. For compounds showing more than $30 \%$ of DPPH scavenging activity from screening test with $100 \mu \mathrm{M}$ concentration in final,

\section{Results and Discussion}

Target compound synthesis. All hydroxydiphenyl ether analogues were prepared by the Ullmann-type ether formation reaction, in which an aryl bromide reacts with phenol under basic conditions in the presence of copper (I) salt to form the intermediate diphenyl ethers. ${ }^{19}$ Synthetic methods applied for the target compounds were depicted in Scheme 1. Guaiacol was coupled with 2-bromonitrobenzene or 2,5-dibromonitrobenzne by Ullmann-type reaction in the presence of copper (I) chloride to obtain compound $\mathbf{1}$ and $\mathbf{2}$. Sodium hydride and $N, N$-dimethylformaide (DMF) were used as a base and solvent, respectively. Demethylation of compound $\mathbf{1}$ and $\mathbf{2}$ was carried out in the presence of $\mathrm{BBr}_{3}$ (2.0 eq.) to afford compound 3 (46\%) and $4(33 \%)$ respectively (Scheme 1$)$. Coupling of 3-methoxyphenol and 2-bromonitrobenzene afforded compound 5 (17\%). In the ${ }^{1} \mathrm{H}$ NMR spectrum, methyl protons of methoxy group of compound $\mathbf{5}$ showed a singlet peak at $\delta 3.79$. Demethylation of 5 under $\mathrm{BBr}_{3}$ condition at RT afforded compounds 6 (58\%), 7 $(11 \%)$ and $8(7 \%)$ together from same reaction mixture.

Using the similar procedure the 3-methoxyphenol treated with 2,5-dibromonitrobenzene to obtain compound 9 (36\%). Subsequent demethylation of 9 with $\mathrm{BBr}_{3}(2.0$ eq.) at $\mathrm{rt}(28 \mathrm{~h})$ afforded compound 10 (15\%) and 11 (44\%).

Coupling reaction of 3,5-dimethoxyphenol and 2-bromonitrobenzene provided compound 12 (12\%). In the ${ }^{1} \mathrm{H}$ NMR spectrum, six protons of two methoxy groups of 12 showed a singlet peak at $\delta 3.74$. Doublet peak $(J=2.0 \mathrm{~Hz})$ of $\mathrm{H}-2$ and $\mathrm{H}-6$ at $\delta$ 6.18 and triplet $(J=2.0 \mathrm{~Hz})$ of $\mathrm{H}-4$ at $\delta 6.26$ were also helped to determine the structure. Demethylation of compound $\mathbf{1 2}$ under $\mathrm{BBr}_{3}$ (1.0 eq.) at rt (24 h) afforded compound 13 (13\%),
$14(8 \%)$ and $15(18 \%)$ at the same time. In the case of compound $14,{ }^{1} \mathrm{H}$ NMR spectrum showed a singlet peak of $\mathrm{H}-2$ and H-6 at $\delta 6.29$ while compound $\mathbf{1 5}$ showed two doublet peaks of H-2 and H-6 at $\delta 6.39(J=2.0 \mathrm{~Hz})$ and $\delta 6.01 \mathrm{ppm}(J=2.0 \mathrm{~Hz})$, respectively.

Coupling reaction of 4-methoxyphenol with 2-bromonitrobenzene or 2,5-dibromonitrobenzene afforded compound $\mathbf{1 6}$ $(15 \%)$ and $17(41 \%)$ respectively. After demethylation under $\mathrm{BBr}_{3}$ condition (1.5 eq) at $\mathrm{rt}(14 \mathrm{~h})$, compound 16 and 17 yielded compound $18(42 \%)$ and $19(82 \%)$ respectively without brominated compounds. All the spectroscopic data were consistent with the expected structures.

Ullmann-type reaction of 3,5-dihydroxyanisole with 2-bromonitrobenzene produced compounds $20(5 \%)$ and $21(6 \%)$ separately in the same reaction mixture. Then compound 22 $(63 \%)$ was obtained by demethylation of compound 20 under $\mathrm{BBr}_{3}$ condition (Scheme 2).

Pharmacological activity test.

Mushroom tyrosinase inhibitory activity: ${ }^{20}$ All the compounds were evaluated for monophenolase inhibitory activity of tyrosinase using L-tyrosine as a substrate. The percentage inhibition is presented in Table 1 and 2. Compound $\mathbf{4}$ showed comparable tyrosinase inhibitory activities to those of arbutin used as a reference within the test concentration range (Table 1). Most other compounds were less active than reference. But, surprisingly, compound 18 and 19 were much stronger inhibitor against tyrosinase function than arbutin (Table 2). Both compounds had para-hydroxyphenyl moiety in their structure. It might be due to their structural resemblance to L-tyrosine, a substrate for tyrosinase during early stage of melanogenesis. These results might reinforce the importance of $p$-hydroxyphenyl group in the tyrosinase inhibitory process, which is consistent with the reported information.

DPPH radical scavenging activity: Diphenyl ethers may exert antioxidant property, due to their ability to donate an electron or chelate metals. Since our compounds also have phenolic hydroxyl group and thus may perform antioxidant activity, we intended to test for anti-oxidative activity possibly to prevent or delay pigmentation resulting from auto-oxidation. The antioxidative activity of selected compounds was tested by measuring their ability to convert DPPH (1,1-diphenyl-2-picryl hydrazyl) free radical to DPPH-H (1,1-diphenyl-2-picryl hydrazine).

Most compounds are inactive to the antioxidant function with DPPH. (Table 3) Unexpectedly, compounds 18 and 19 were also lack of scavenging activity. This result showed the discrepancy between the antityrosinase activity and DPPH radical scavenging activity among the compounds tested. Compounds 13, 14 and $\mathbf{1 5}$ possessing resorcinol moiety in the structure exhibited moderate antioxidant activity but lower than ascorbic acid used as positive control. Because of law potency of the compounds $\mathrm{EC}_{50}$ of the compounds were not determined. This information implies that elaborate calibration of the resorcinol portion of diphenyl ether can increase the antioxidant ability of the parent compounds.

\section{Conclusions}

We have synthesized 14 diphenyl ether compounds possess- 
<smiles>COc1ccccc1O</smiles><smiles>[R]c1ccc(Br)c([N+](=O)[O-])c1</smiles>

$\mathrm{R}=\mathrm{H}, \mathrm{Br}$

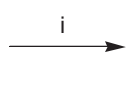

$\mathrm{H}_{3} \mathrm{COH}+$<smiles>O=[N+]([O-])c1ccccc1Br</smiles>

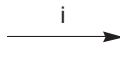<smiles>COc1cccc(Oc2ccccc2[N+](=O)[O-])c1</smiles>

5
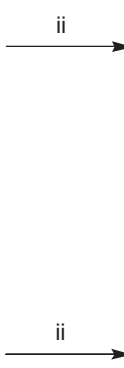<smiles>COc1cccc(Oc2ccc(Br)cc2[N+](=O)[O-])c1</smiles>

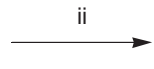<smiles>O=[N+]([O-])c1cccc(Oc2ccccc2O)c1</smiles>

$3 \mathrm{R}=\mathrm{H}$
$\mathrm{R}=\mathrm{Br}$<smiles>[R]c1cc([R])c(Oc2ccccc2[N+](=O)[O-])cc1O</smiles>

$6 \mathrm{R}_{1}=\mathrm{H}, \mathrm{R}_{2}=\mathrm{H}$

$7 \mathrm{R}_{1}=\mathrm{Br}, \mathrm{R}_{2}=\mathrm{H}$

$8 \mathrm{R}_{1}=\mathrm{H}, \mathrm{R}_{2}=\mathrm{Br}$<smiles>COc1cccc(O)c1</smiles><smiles>[R]c1ccc(O)cc1Oc1ccc(Br)cc1[N+](=O)[O-]</smiles>

$10 \mathrm{R}=\mathrm{Br}$ $11 \mathrm{R}=\mathrm{H}$<smiles>[R]c1c(O)cc(Oc2ccccc2[N+](=O)[O-])c([R2])c1O</smiles>

$3 \mathrm{R}_{1}=\mathrm{H}, \mathrm{R}_{2}=\mathrm{H}$

$14 \mathrm{R}_{1}=\mathrm{Br}, \mathrm{R}_{2}=\mathrm{H}$ $15 \mathrm{R}_{1}=\mathrm{H}, \mathrm{R}_{2}=\mathrm{Br}$<smiles>[R]c1ccc(Br)c([N+](=O)[O-])c1</smiles>
$\mathrm{R}=\mathrm{H}, \mathrm{Br}$

12

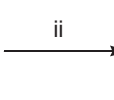<smiles>COc1cc(OC)cc(Oc2ccccc2[N+](=O)[O-])c1</smiles><smiles>[R]c1ccc(Oc2ccc(OC)cc2)c([N+](=O)[O-])c1</smiles><smiles>C=CC</smiles><smiles>[R]c1ccc(Oc2ccc(O)cc2)c([N+](=O)[O-])c1</smiles>

$18 \mathrm{R}=\mathrm{Br}$

Scheme 1. Synthesis of compounds. Reagents and conditions: (i) $\mathrm{CuCl}, \mathrm{NaH}, \mathrm{DMF}$, reflux; (ii) $\mathrm{BBr}_{3}, \mathrm{ClCH}_{2} \mathrm{CH}_{2} \mathrm{Cl}_{\text {, } \mathrm{rt}}$<smiles>COc1cc(O)cc(O)c1</smiles><smiles>COc1cc(Oc2ccccc2[N+](=O)[O-])cc(Oc2ccccc2[N+](=O)[O-])c1</smiles>

20<smiles>COc1cc(O)cc(Oc2ccccc2[N+](=O)[O-])c1</smiles>

21

Scheme 2. Synthesis of compounds. Reagents and conditions: (i) $\mathrm{CuCl}, \mathrm{NaH}, \mathrm{DMF}$, reflux; (ii) $\mathrm{BBr}_{3}, \mathrm{ClCH}_{2} \mathrm{CH}_{2} \mathrm{Cl}, \mathrm{rt}$ 
Table 1. Tyrosinase inhibitory activity (\%) of compounds

\begin{tabular}{cccc}
\hline \multirow{3}{*}{ Group } & \multicolumn{3}{c}{ Dose $(\mu \mathrm{g} / \mathrm{mL})$} \\
\cline { 2 - 4 } & 10 & 20 & 40 \\
\hline $\mathbf{3}$ & $4.97 \pm 0.48$ & $8.02 \pm 0.71$ & $9.04 \pm 0.13$ \\
$\mathbf{4}$ & $23.72 \pm 1.99$ & $40.58 \pm 1.53$ & $60.93 \pm 1.94$ \\
$\mathbf{6}$ & $4.95 \pm 0.38$ & $6.88 \pm 0.50$ & $7.91 \pm 0.27$ \\
$\mathbf{7}$ & $23.07 \pm 2.49$ & $41.17 \pm 1.75$ & $61.92 \pm 1.56$ \\
$\mathbf{8}$ & $11.42 \pm 1.57$ & $12.47 \pm 0.64$ & $16.80 \pm 0.50$ \\
$\mathbf{1 0}$ & $25.38 \pm 2.27$ & $38.2 \pm 1.18$ & $46.92 \pm 0.58$ \\
$\mathbf{1 1}$ & $5.32 \pm 0.21$ & $9.12 \pm 0.83$ & $14.52 \pm 0.44$ \\
$\mathbf{1 3}$ & $3.47 \pm 0.64$ & $7.04 \pm 0.59$ & $7.73 \pm 0.22$ \\
$\mathbf{1 4}$ & $0.60 \pm 0.29$ & $6.32 \pm 1.26$ & $8.23 \pm 0.11$ \\
$\mathbf{1 5}$ & $6.58 \pm 0.94$ & $17.39 \pm 1.85$ & $24.58 \pm 0.46$ \\
$\mathbf{2 1}$ & 0 & 0 & 0 \\
$\mathbf{2 2}$ & $6.68 \pm 0.24$ & $17.53 \pm 2.40$ & $27.28 \pm 1.00$ \\
Arbutin & $49.25 \pm 0.75$ & $68.63 \pm 1.09$ & $81.46 \pm 1.57$ \\
\hline
\end{tabular}

Values represent means \pm S.D. $(n=3)$.

Table 2. Tyrosinase inhibitory activity (\%) of compound $\mathbf{1 8}$ and 19

\begin{tabular}{cccc}
\hline \multirow{3}{*}{ Compound } & \multicolumn{3}{c}{ Dose $(\mu \mathrm{g} / \mathrm{mL})$} \\
\cline { 2 - 4 } & 1 & 5 & 10 \\
\hline $\mathbf{1 8}$ & $42.60 \pm 0.90$ & $57.84 \pm 1.61$ & $71.27 \pm 2.20$ \\
$\mathbf{1 9}$ & $26.21 \pm 2.0$ & $73.77 \pm 1.25$ & $82.67 \pm 0.39$ \\
Arbutin & $\mathrm{NA}^{a}$ & $\mathrm{NA}$ & $49.25 \pm 0.75$ \\
\hline
\end{tabular}

Values represent means \pm S.D. $(n=3) .{ }^{a}$ Not analyzed.

Table 3. DPPH scavenging activities of compounds

\begin{tabular}{ccc}
\hline Compound & DPPH scavenging activity $(\%)^{a}$ & $\mathrm{EC}_{50}(\mu \mathrm{M})^{b}$ \\
\hline Vitamin C & 93.90 & $26.22 \pm 1.45$ \\
$\mathbf{3}$ & 0 & $\mathrm{NA}^{c}$ \\
$\mathbf{4}$ & 7.28 & $\mathrm{NA}^{c}$ \\
$\mathbf{6}$ & 3.00 & $\mathrm{NA}^{c}$ \\
$\mathbf{7}$ & 8.08 & $\mathrm{NA}^{c}$ \\
$\mathbf{8}$ & 0 & $\mathrm{NA}^{c}$ \\
$\mathbf{1 0}$ & 7.57 & $\mathrm{NA}^{c}$ \\
$\mathbf{1 1}$ & 0.86 & $\mathrm{NA}^{c}$ \\
$\mathbf{1 3}$ & 28.79 & $\mathrm{NA}^{c}$ \\
$\mathbf{1 4}$ & 45.10 & $\mathrm{NA}^{c}$ \\
$\mathbf{1 5}$ & 17.01 & $\mathrm{NA}^{c}$ \\
$\mathbf{1 8}$ & 11.68 & $\mathrm{NA}^{c}$ \\
$\mathbf{1 9}$ & 3.68 & $\mathrm{NA}^{c}$ \\
$\mathbf{2 1}$ & 7.54 & $\mathrm{NA}^{c}$ \\
$\mathbf{2 2}$ & 4.97 & $\mathrm{NA}^{c}$ \\
\hline
\end{tabular}

$\overline{{ }^{a}}$ The percent DPPH scavenging activity was obtained with $100 \mu \mathrm{M}$ concentration of test compounds. ${ }^{b}$ Concentration for scavenging $50 \%$ of DPPH free radical was expressed in mean value \pm standard deviation obtained in triplicate. ${ }^{c}$ Not analyzed. ing hydroxyl, bromo, and nitro groups in the structure. Compounds 18 and 19 have shown much stronger inhibition of tyrosinase monophenolase function than arbutin used as a positive control. Both compounds possess para-hydroxyphenyl moiety in their structure, which might reinforce the importance of $p$-hydroxyphenyl group in the tyrosinase inhibitory process. In the DPPH radical scavenging activity test, none of the compounds even 18 and 19 showed significant antioxidant activity. The result obtained suggests that elaborate adjustment of diphenyl ether analogues with proper substituents may have potential to be developed as new skin whitening agents working on the tyrosinase function.

Acknowledgments. This work was support by the RIC program of MKE (Ministry of Knowledge Economy), Korea.

\section{References}

1. Schallreuter, K. U.; Kothari, S.; Chavan, B.; Spencer, J. D. Exp. Dermatol. 2007, 17, 395.

2. Parvez, S.; Kang, M.; Chung, H. S.; Bae, H. Phytother. Res. 2007, $21,805$.

3. Criton, M.; Le Mellay-Hamon, V. Bioorg. Med. Chem. Lett. 2008, 12,3607 .

4. Hearing, V. J.; Tsukamoto, K. FASEB. J. 1991, 5, 2902.

5. Likhitwitayawuid, K. Curr. Sci. 2008, 94, 44.

6. Kim, Y. J.; No, J. K.; Lee, J. H.; Chung, H. A. Biol. Pharm. Bull. 2005, 28, 323.

7. Solano, F.; Briganti, S.; Picardo, M.; Ghanem, G. Pigment Cell Res. 2006, 19, 550.

8. Menter, J. M.; Etemadi, A. A.; Chapman, W.; Hollins, T. D.; Willis, I. Melanoma Res. 1993, 3, 443.

9. Khatib, S.; Nerya, O.; Musa, R.; Shmuel, M.; Tamir, S.; Vaya, J. Bioorg. Med. Chem. 2005, 13, 433.

10. Passi, S.; Nazzaro-Porro, M. Br. J. Dermatol. 1981, 104, 659.

11. Briganti, S.; Camera, E.; Picardo, M. Pigment Cell Res. 2003, $16,101$.

12. Ohyama, Y.; Mishima, Y. Fragrance J. 1990, 6, 53.

13. a) Tokiwa, Y.; Kitagawa, M.; Raku, T.; Yanagitani, S.; Yoshino, K. Biorg. Med. Chem. Lett. 2007, 17, 3105. b) Hori, I.; Nihei, K.-I.; Kubo, I. Phytother. Res. 2004, 18, 475.

14. Oozeki, H.; Tajima, R.; Nihei, K.-i. Bioorg. Med. Chem. Lett. 2008, 18, 5252.

15. Chomcheon, P.; Wiyakrutta, S.; Sriubolmas, N.; Ngamrojanavanich, N.; Kengtong, S.; Mahidol, C.; Ruchirawat, S.; Kittakoop, P. Phytochem. 2009, 70, 407.

16. Marcoux, J.-F.; Doye, S.; Buchwald, S. L. J. Am. Chem. Soc. 1997, $119,10539$.

17. Xu, H.; Jian, K.-Z.; Guan, Q.; Ye, F.; Lv, M. Chem. Pharm. Bull. 2007, 55, 1755.

18. Li, K.; Li, X.-M.; Ji, N.-Y.; Wang, B.-G. Bioorg. Med. Chem. 2007 , 15,6627 .

19. de la Fuente, J. A.; Manzanaro, S.; Martin, M. J.; de Quesada, T. G.; Reymundo, I.; Leungo, S. M.; Gago, F. J. Med. Chem. 2003, 46, 5208.

20. Masamoto, Y.; Ando, H.; Murata, Y.; Shimoishi, Y.; Tada, M.; Tanahata, K. Biosci. Biotechnol. Biochem. 2003, 67, 631.

21. Kim, B.-T.; O, K.-J.; Chun, J.-C.; Hwang, K.-J. Bull. Korean Chem. Soc. 2008, 29, 1125. 